and trainee rheumatologists who enrolled patients in these studies, and all research nurses for their contributions.

Disclosure of Interests: Johanna M. Maassen: None declared, Raquel Dos-Santos: None declared, Sytske Anne Bergstra: None declared, Robbert Goekoop: None declared, Thomas Huizinga: None declared, Cornelia Allaart Grant/research support from: The original BeSt study was realized with a government grant from the Dutch College of Health Insurance Companies, with additional funding from Schering-Plough and Janssen. the IMPROVED study was financially supported by AbbVie in the first year

DOI: 10.1136/annrheumdis-2021-eular.2442

\section{POS0471 ASSOCIATION OF HLA-DRB1 HAPLOTYPES WITH CARDIOVASCULAR MORTALITY IN INFLAMMATORY POLYARTHRITIS: RESULTS FROM THE NORFOLK ARTHRITIS REGISTER}

S. Sharma ${ }^{1}$, D. Plant ${ }^{1}$, J. Bowes ${ }^{1}$, A. MacGregor ${ }^{2,3}$, S. Verstappen ${ }^{1,4}$, A. Barton ${ }^{1,4}$, S. Viatte ${ }^{1,5}$. ${ }^{1}$ Centre for Genetics and Genomics Versus Arthritis, Centre for Musculoskeletal Research, Manchester, United Kingdom; ${ }^{2}$ Norwich Medical School, University of East Anglia Faculty of Medicine and Health Sciences, Norwich, United Kingdom; ${ }^{3}$ Rheumatology Department, Norfolk and Norwich University Hospitals NHS Foundation Trust, Norwich, United Kingdom; ${ }^{4}$ NIHR Manchester Musculoskeletal Biomedical Research Unit, Central Manchester NHS Foundation Trust, Manchester, United Kingdom; ${ }^{5}$ Lydia Becker Institute of Immunology and Inflammation, Faculty of Biology, Medicine and Health; The University of Manchester, Manchester, United Kingdom

Background: Haplotypes defined by amino acids at HLA-DRB1 positions 11, 71 and 74 associated with susceptibility to rheumatoid arthritis (RA), are associated with radiological outcome, anti-TNF response and all cause-mortality in RA.(1, 2) RA is associated with cardiovascular (CV) morbidity and mortality, but the increased prevalence of risk factors of CV disease in RA only partially explains this association.

Objectives: The aim was to investigate whether haplotypes associated with RA disease susceptibility and disease severity are also associated with CV mortality. Methods: The Norfolk Arthritis register (NOAR) is a primary care-based inception cohort of patients with inflammatory polyarthritis (IP). $(1,2)$ NOAR patients with at least 2 years of follow-up and available mortality and genetic data were included in this study. Mortality data was provided by the Office for National Statistics.

Univariate Cox proportional hazard models were applied using STATA/IC 14.0. Models for CV mortality were adjusted for CV risk factors selected using stepwise regression: namely obesity, gender and hypertension. Hazard models were applied to the entire cohort of patients with inflammatory polyarthritis (IP). When calculating differences between highest and lowest risk genetic factors, bivariate analysis was used.

Results: HLA-DRB1 amino acids, haplotypes or haplotype groups associated with RA susceptibility are also associated with CV mortality as shown in the table 1. HLA-DRB1 polymorphisms encoding amino-acid haplotypes associated with an increased or decreased susceptibility to RA consistently show the same magnitude and direction of association for overall and CV mortality in IP. For example, the SEA-haplotype, associated with the lowest susceptibility to RA, and the best radiographic outcome, was found to be associated with decreased CV mortality (HR 0.67, 95\% $\mathrm{Cl} 0.47,0.91, \mathrm{p}=0.023$ ). The relative difference in $\mathrm{CV}$ mortality between carriers of the high susceptibility VKA haplotype and carriers of the SEA haplotype was significant (HR 1.67, 95\% Cl 1.13, 2.48, $p=0.01)$.

Table 1. Hazard ratios for CV mortality by genetic factors among patients with IP. Total number ( $n$ ) of deaths are also displayed alongside the total number $(n)$ of patients included in each analysis.

\begin{tabular}{lll}
\hline Amino acid / Haplotype /Group & Hazard Ratio $(95 \% \mathrm{Cl})$ & $\mathrm{N}$ \\
\hline valine 11 & $1.10(0.93,1.30)$ & $343(2514)$ \\
serine 11 & $0.82(0.70,0.96)^{\star}$ & $343(2514)$ \\
Difference & $1.23(1.01,1.49)^{*}$ & \\
VKA haplotype & $1.16(0.94,1.43)$ & $310(2328)$ \\
SEA haplotype & $0.67(0.47,0.94)^{*}$ & $310(2328)$ \\
Difference & $1.67(1.13,2.48)^{*}$ & $319(2328)$ \\
Group $1^{\dagger}$ & $1.10(0.93,1.31)$ & $319(2328)$ \\
Group 4 & &
\end{tabular}

${ }^{*} p<0.05+$ Haplotype groups as defined previously (2)

HLA-DRB1 haplotypes can be ranked according to the magnitude of their association with RA susceptibility and this hierarchy is conserved for various measures of disease outcome and overall mortality. $(2,3)$ The figure 1 shows that this risk hierarchy is also conserved for CV mortality: HLA-DRB1 haplotypes that predispose to RA also predispose to increased CV mortality, independent of known $\mathrm{CV}$ risk factors.

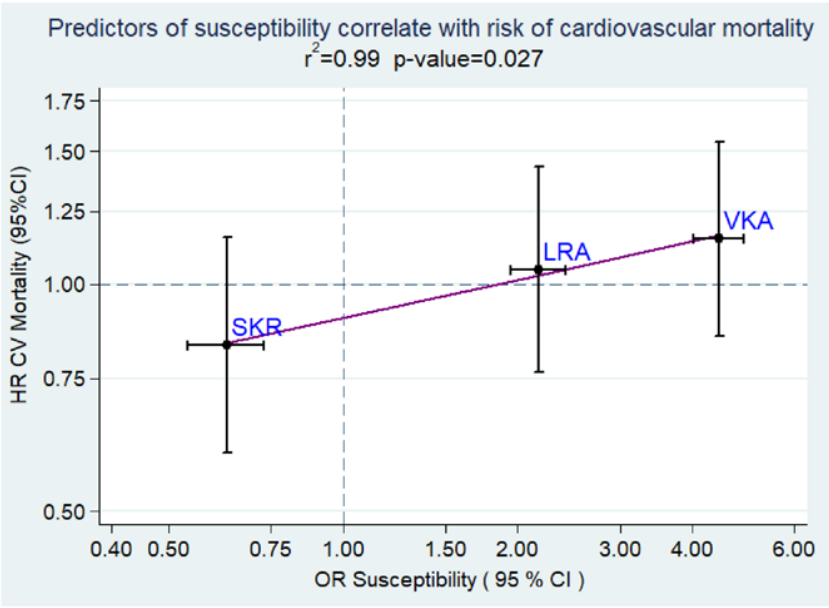

Figure 1. Haplotypes with frequency $>12 \%$ in NOAR are shown. $X$ axis: susceptibility to ACPA-positive RA as ORs.(2) Y axis: CV mortality risk in IP as HRs, derived from multi-variate cox-proportional hazard models adjusted for $\mathrm{CV}$ risk factors. Values are on a logarithmic scale. A one-tailed $p$ value was calculated using linear regression to determine the association between $\beta$ coefficients of susceptibility and CV mortality.

Conclusion: The originally reported genetic associations between HLA-DRB1 polymorphisms and overall mortality were likely driven by associations with $\mathrm{CV}$ mortality.

\section{REFERENCES:}

[1] S. Viatte et al., JAMA 313, 1645-1656 (2015).

[2] S. Raychaudhuri et al., Nat Genet 44, 291-296 (2012).

[3] D. P. Symmons, A. J. Silman, Clin Exp Rheumatol 21, S94-99 (2003).

Disclosure of Interests: None declared

DOI: 10.1136/annrheumdis-2021-eular.248

\section{POS0472 COMPARATIVE METABOLOMIC ANALYSIS OF SERUM SAMPLES FROM PATIENTS WITH COINCIDENTAL RHEUMATOLOGICAL AND MALIGNANT DISEASES}

D. Marx ${ }^{1}$, L. Diekmann ${ }^{1}$, K. Klika ${ }^{2}$, H. M. Lorenz ${ }^{1}$, K. Benesova ${ }^{1}$, M. SoutoCarneiro'. ' Heidelberg University Hospital, Medicine 5 - Rheumatology, Heidelberg, Germany; ${ }^{2}$ DKFZ Heidelberg, Molecular Structure Analysis, Heidelberg, Germany

Background: Rheumatic and musculoskeletal diseases (RMDs) and malignancies are both caused by a dysfunctional immune system and the probability of their coincidence in one individual is rising due to advances in cancer treatment and demographic changes. However, the lack of understanding of the complex interrelationship of both conditions often leads to undertreatment and high level of suffering in affected patients. Herein, the MalheuR project breaks new ground by systematic analysis of concomitant malignant and rheumatic diseases and closes the knowledge gaps on the clinical and molecular level.

Objectives: To enable early diagnosis of concomitant malignancy and/or identification of patients at risk in the future, changes in serum metabolome were explored in order to create a diagnostic classification model.

Methods: Serum samples from patients with concomitant RMD and cancer or obligate precancerous lesions ( $n=78$, breast cancer (23), melanoma (14), MGUS (12), prostate cancer (8) and others (21)) were collected as a pilot study within the MalheuR project, a registry-based study initiated in 2018 at the university hospital Heidelberg, Germany. The following groups were defined by the underlying RMD: rheumatoid arthritis $(n=42)$, psoriasis arthritis $(n=23)$, spondylarthritis $(n=9)$ and systemic lupus erythematosus $(n=4)$. RMD patients without any malignancies were used as controls ( $n=280$ : 122 RA, 81 PsA, 46 SpA, 31 SLE).

Samples were analyzed by $1 \mathrm{H}$ NMR spectroscopy. For all samples, regular $1 \mathrm{H}$ acquisition with presaturation and Carr-Purcell-Meiboom-Gill (CPMG) spectra were acquired using a $600 \mathrm{MHz}$ Bruker NMR spectrometer. Spectra were processed with TopSpin using $0.2 \mathrm{~Hz}$ of line broadening and manual phasing. Molar concentrations of 26 metabolites were acquired by integration of NMR spectra. With GraphPad Prism, univariate and ANOVA statistical analysis was performed to find significant differences between each malignant group and their control group as well as between all four malignant groups. 
Results: Mean disease duration was $11.8 \pm 10.5$ years for cancer and $12.8 \pm 10.8$ years for RMDs since diagnosis. $1.4 \%$ received cancer treatment $(6.4 \%$ of malignancy group), $69.3 \%$ csDMARDs, $42.3 \%$ b/tsDMARDs and $46.4 \%$ glucocorticoids at the time of sample collection.

Most metabolites tested were significantly lower in the malignancy groups versus associated controls: Concentrations of amino acids $V$ and $L$ were significantly reduced in all malignancy samples. Additionally, T, D, N, Q, E, A, I were altered in RA, SpA and PsA, changes in G were seen in RA, PsA and SLE and $P$ was altered in RA and PsA only. Furthermore, lower concentrations of short chain fatty acids and tricarboxylic acid cycle intermediates were present in the malignancy groups. In no case was a metabolite concentration significantly higher in the malignancy group than in the associated control. When comparing the metabolome within the four malignancy groups, only the concentrations of creatine, threonine and isoleucine were found higher in RA patients with malignancy.

Conclusion: Significant differences between the metabolomic fingerprints of RMD patients with and without malignancies could be observed. These changes might be characteristic for cancer burden, as in most cases the underlying RMD was not relevant when comparing the concentrations between the malignancy groups. Our results may promote understanding of the interrelationships of both disease entities as well as prove useful as biomarkers for diagnostic and therapeutic purposes.

Acknowledgements: Grant/research support from medical faculty (Olympia Morata Programme) and foundations commission (Herbert Daus estate) of University of Heidelberg

Disclosure of Interests: None declared

DOI: 10.1136/annrheumdis-2021-eular.2484

\section{POS0473 AUTOIMMUNITY AGAINST COLLAGEN IN RHEUMATOID ARTHRITIS (RA): SERUM LEVELS OF IGG AGAINST DENATURED TYPE II COLLAGEN SIGNIFICANTLY ELEVATED IN RA}

P. Drobinski ${ }^{1}$, E. Aighobahi ${ }^{2}$, A. C. Bay-Jensen ${ }^{3}$, M. Karsdal ${ }^{3} .{ }^{1}$ University of Copenhagen/Nordic Bioscience, Department of Biomedical Sciences, Copenhagen, Denmark; ${ }^{2}$ Technical University of Denmark, Department of Biotechnology and Biomedicine, Copenhagen, Denmark; ${ }^{3}$ Nordic Bioscience, ImmunoScience, Herlev, Denmark

Background: The presence of autoantibodies in Rheumatoid Arthritis (RA) is a hallmark of the disease and one of main criteria for diagnosis and clinical classification. Identification of anti-citrullinated protein antibodies (ACPA) and RF-Factors in circulation of RA patients remains the most characteristic and established criteria for diagnosis. However, due to diagnostic limitation of these biomarkers, only approximately $70 \%$ of patients with RA can be identified. As a consequence, there is a lack of diagnostic biomarkers for large number of patients. Diagnosis of these patients relies mainly on assessment of their clinical symptoms such as swollen and tender joints. This problem leaves a need for diagnostic improvement and development of more sensitive biomarkers.

Objectives: In this study, we aimed to develop a sensitive biomarker assay capable to identify and quantify presence of autoantibodies against denatured type II collagen in circulation of patients with RA.

Methods: The presence and levels of autoantibodies was measured in serum samples from 50 patients with moderate to severe RA who had inadequate response to Methotrexate (average age $51.2,86 \%$ of female, $78 \%$ of white race patients). Control cohort involved serum from 42 healthy age matched patients (average age $48.6,50 \%$ of female, $60 \%$ of white race patients). Denaturation of the type II collagen was performed by heat treatment for $15 \mathrm{~min}$ at $72{ }^{\circ} \mathrm{C}$. Improvement of assay sensitivity was investigated by measurements of autoimmunity levels against denatured and native type II collagen. Assay specificity was assessed by comparison of presence of autoantibodies against type II collagen versus albumin (non-sense control). The normality of data distribution was checked with Shapiro-Wilk test, significance between cohorts with Mann-Whitney nonparametric test and correlations with Spearman's correlation coefficient (rho).

Results: Serum levels of autoantibodies against denatured type II collagen were significantly higher in RA patients than in healthy controls $(P<0.0001)$ (Figure 1). We observed nearly 4 -fold difference between both cohorts. Denaturation of type II collagen showed high improvement of assay sensitivity and increased accessibility of collagen for binding of autoantibodies. Developed assay showed specificity for detection of type II collagen autoantibodies by displaying no levels of autoimmunity against other control proteins (albumin). The levels of serum autoantibodies correlated significantly $(P<0.03)$ with patient disease activity (DAS28) at baseline, displaying rho $=0.3$. Our assay showed good technical performance with acceptable inter- and intra- assay variations (18\% and $5 \%$ respectively).

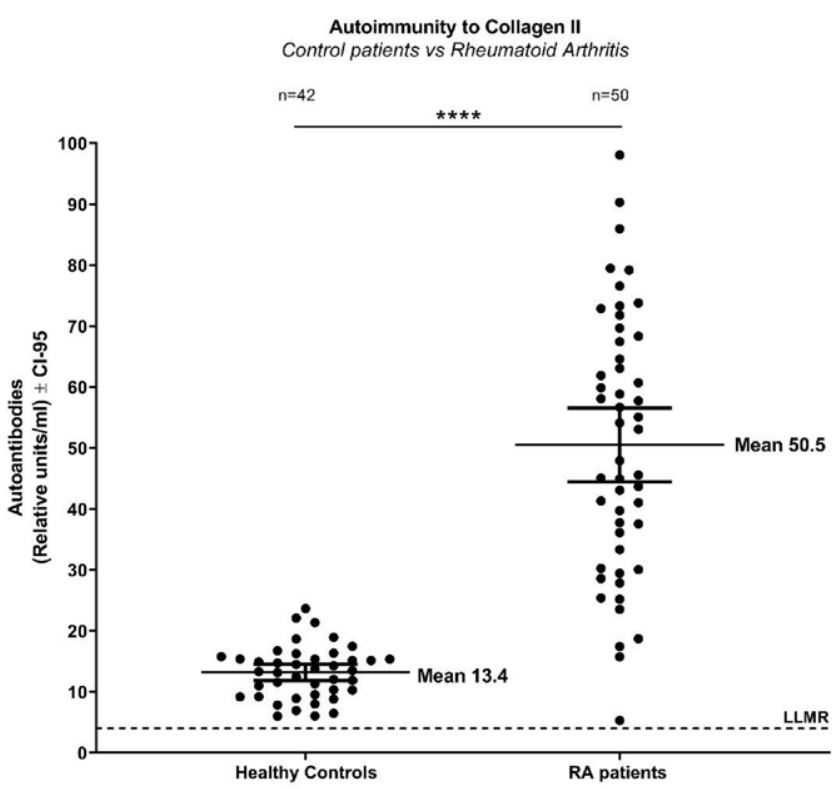

Figure 1. Differences in serum levels of autoantibodies against denatured type II collagen between healthy controls and patients with Rheumatoid Arthritis.

Conclusion: Present findings show that patients with RA carry upregulated levels of circulating autoantibodies directed against type II collagen. Heat-treatment of type II collagen increased exposure of immunogenic epitopes of collagen and enabled for more sensitive detection of autoantibodies directly in patient serum. Developed assay demonstrated potential for specific detection of autoantibodies and may provide additional diagnostic value in RA patients.

Disclosure of Interests: Patryk Drobinski: None declared, Elijah Aighobahi: None declared, Anne-Christine Bay-Jensen Shareholder of: Nordic Bioscience A/S, Employee of: Nordic Bioscience A/S, Morten Karsdal Shareholder of: Nordic Bioscience A/S, Employee of: Nordic Bioscience A/S DOI: 10.1136/annrheumdis-2021-eular.2488

\begin{tabular}{|l|l}
\hline POS0474 & SUBSTANTIAL IMPACT OF AUTOANTIBODY \\
& ENRICHMENT ON OUTCOMES IN EARLY \\
& RHEUMATOID ARTHRITIS TREATED WITH \\
& ABATACEPT: DATA FROM A LARGE POOLED \\
& ANALYSIS OF 4 RCTS
\end{tabular}

K. Michaud $^{1,2}$, S. S. H. Park ${ }^{3}$, K. Lozenski ${ }^{4}$, J. Vaile ${ }^{5}$, V. Khaychuk ${ }^{6}$, J. Pope ${ }^{7}$, P. G. Conaghan ${ }^{8,9} .{ }^{1}$ University of Nebraska Medical Center, Internal Medicine Division of Rheumatology, Omaha, NE, United States of America; ${ }^{2}$ FORWARD, The National Data Bank for Rheumatic Diseases, Wichita, KS, United States of America; ${ }^{3}$ Bristol-Myers Squibb, US HEOR, Princeton, NJ, United States of America; ${ }^{4}$ Bristol-Myers Squibb, Medical Data Generation, Princeton, NJ, United States of America; ${ }^{5}$ Bristol-Myers Squibb, Worldwide Medical, Princeton, NJ, United States of America; ${ }^{6}$ Bristol-Myers Squibb, US Medical Immunology and Fibrosis, Princeton, NJ, United States of America; ${ }^{7}$ Western University, Schulich School of Medicine, Division of Rheumatology, London, Ontario, Canada: ${ }^{8}$ University of Leeds, Institute of Rheumatic and Musculoskeletal Medicine, Leeds, United Kingdom; ${ }^{9}$ Leeds Teaching Hospitals NHS Trust, NIHR Leeds Biomedical Research Centre, Leeds, United Kingdom

Background: Biomarkers are commonly used as prognostic tools in rheumatoid arthritis (RA) and have additional potential to guide treatment decisions. Previous clinical trials of abatacept (ABA) such as the Early AMPLE trial (eAMPLE) and real-world data suggest differential treatment effects of $A B A$ based on the presence of biomarkers and disease stage..$^{1-4}$

Objectives: To supplement evidence of enhanced efficacy of ABA among patients with enriched autoantibody biomarkers and early disease stage by applying key inclusion criteria from the eAMPLE trial retrospectively to historic ABA RCTs.

Methods: Individual patient data (IPD) from four early-RA ABA trials (AGREE [NCT00122382], AMPLE [NCT00929864], AVERT [NCT01142726], AVERT-2 [NCT02504268]) were pooled. Patients were defined as enriched at baseline if they had: 1) RA disease duration $\leq 12$ months, 2) DAS28-CRP $\geq 3.2,3$ ) $\geq 3$ times the upper limit of normal on an anti-cyclic citrullinated peptide (anti-CCP) test, and 4$)$ were rheumatoid factor positive $(R F+)$. Patients were grouped according to treatment status (ABA [monotherapy or with methotrexate (MTX)] or non-ABA [MTX or adalimumab (ADA) plus MTX]). Outcomes included DAS28-CRP mean 